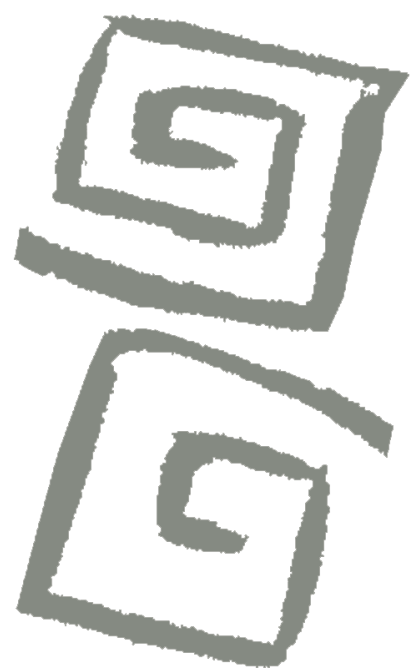

\title{
Prevenir la obesidad en contextos de precarización: respuestas locales a estrategias globales
}

\author{
Preventing obesity in precarious contexts: local \\ responses to global strategies
}

Mabel Gracia Arnaiz ${ }^{1}$, Flavia Demonte ${ }^{2}$, Fabiana Bom Kraemer ${ }^{3}$

\author{
${ }^{1}$ Autora de correspondencia. \\ Doctora en Antropología \\ Social. Catedrática de \\ Antropología Social, \\ Universitat Rovira i Virgili, \\ Tarragona, España. $\bowtie$ (iD) \\ ${ }^{2}$ Doctora en Ciencias \\ Sociales. Investigadora \\ Adjunta, Consejo Nacional \\ de Investigaciones Científicas \\ y Técnicas, con sede en \\ Instituto de Altos Estudios \\ Sociales, Universidad \\ Nacional de San Martín, \\ Buenos Aires, Argentina. \\ $\triangle$ (iD) \\ ${ }^{3}$ Doctora en Ciencias. \\ Profesora Adjunta, \\ Universidade do Estado \\ de Río de Janeiro, Rio de \\ Janeiro, Brasil. $\square$ iD
}

RESUMEN El objetivo de este artículo es analizar la estructura conceptual subyacente de los modelos preventivos sobre la obesidad implementados en Argentina, Brasil y España. En un contexto culturalmente distinto, pero epidemiológicamente similar, los tres países han diseñado sus estrategias, reproduciendo el diagnóstico global sobre sus causas y replicando algunas de las medidas propuestas a nivel mundial. Mientras que los denominados "ambientes obesogénicos" son considerados los principales responsables de estas tendencias, las acciones de educación alimentaria y nutricional promueven la autovigilancia y la racionalización de las prácticas como herramienta principal para lograr cambios en la dieta y la actividad física. Aunque las medidas propuestas a nivel local han sido variadas, fueron menos plurales en cuanto a su naturaleza y alcance, y apenas se incorporan las limitaciones que dificultan adoptar estilos de vida saludables. En contextos de precarización social y alimentaria, esto ha supuesto desconsiderar a los grupos sociales con mayor prevalencia de obesidad.

PALABRAS CLAVES Obesidad; Programas Preventivos; Estrategias Mundiales; Política Nacional de Salud.

\begin{abstract}
The aim of this article is to analyze the conceptual structure underlying the models of obesity prevention implemented in Argentina, Brazil, and Spain. In their culturally distinct but epidemiologically similar contexts, the three countries have devised strategies that reproduce global diagnoses of the causes of obesity and replicate some of the measures proposed at the global level. While so-called "obesogenic environments" are considered primarily responsible for these tendencies, efforts to raise awareness about food and nutrition tend to promote self-monitoring and behavior rationalization as the main tools for achieving changes in diet and physical activity. Although a variety of measures have been proposed at the local level, they have been less diverse in terms of their nature and scope, barely taking into account the constraints that hinder the adoption of healthy lifestyles. In contexts of social and food precarity, this has meant neglecting the social groups with the highest prevalence of obesity.
\end{abstract}

KEY WORDS Obesity; Preventive Health Services; World Strategies; Health Policy. 


\section{INTRODUCCIÓN}

Desde que la obesidad fue declarada epidemia mundial por la Organización Mundial de Salud $(\mathrm{OMS})^{(1,2)}$, y caracterizada por algunos autores como "globesidad"(3) se implementaron diferentes medidas para prevenirla y controlarla. A partir de su crecimiento estadístico, la obesidad se construye epistemológicamente como enfermedad ${ }^{(4)}$, es clasificada por los especialistas como crónica no transmisible y asociada con factores biológicos, conductuales, culturales y ambientales. ${ }^{(5)}$ Dadas las dificultades relacionadas con su prevención y tratamiento ${ }^{(6)}$ y su creciente prevalen$\mathrm{cia}^{(7,8)}$ los factores ambientales han adquirido un mayor poder explicativo, ocupando un lugar estratégico en su definición y en las propuestas de intervención ${ }^{(9)}$. Se la considera, entonces, como la consecuencia directa de dietas hipercalóricas, producto del aumento del consumo de alimentos ultraprocesados y de gastos energéticos insuficientes como expresión última del actual sistema alimentario agroindustrial y de la mecanización de la vida cotidiana $^{(10,11,12)}$ en el marco de sociedades contemporáneas consideradas como "obesogénicas" o ambientalmente "tóxicas"

En este contexto, y formando parte de un proyecto de mayor alcance titulado "La precarización de la vida cotidiana: (in)seguridad alimentaria, género y salud", cuya dirección estuvo a cargo de la Dra. Mabel Gracia Arnaiz, en el presente artículo analizamos la estructura conceptual subyacente de los modelos preventivos sobre la obesidad, implementados en España, Argentina y Brasil entre 2004-2019 y reflexionamos sobre las razones que explicarían la relativa eficacia de estas medidas. Si bien en un estudio anterior se ha analizado el caso específico de España ${ }^{(15)}$, en este hemos querido indagar comparativamente las características de los modelos preventivos y averiguar si estos han tenido en cuenta los cambios socioeconómicos que han acontecido en los países seleccionados. Aunque reconocemos que las características culturales, políticas y económicas son diferentes, España, Argentina y Brasil comparten algunas características que han facilitado la comparación. La preocupación por el aumento sostenido de la obesidad ${ }^{(16,17)}$ coincide, paradójicamente, con el incremento de la aplicación de protocolos de diagnóstico y tratamiento clínico, con la implementación de numerosas acciones preventivas y, también, con que sus respectivas poblaciones parecen tener mayores conocimientos sobre alimentación saludable. Asimismo, la mayor parte de las medidas se han implementado a la par del impacto de las últimas crisis económicas que han llevado al empobrecimiento de una parte significativa de la población de estos países.

Partiendo de la constatación de que las medidas adoptadas no han conseguido hasta la fecha frenar $y / o$ invertir la prevalencia de la obesidad, particularmente entre las personas en situación de mayor precarización social y alimentaria, partimos de la hipótesis de que ello podría estar relacionado con la misma estructura conceptual de los modelos preventivos, los cuales -aun enfatizando la relevancia de los entornos- han puesto excesivo énfasis en la responsabilidad individual al omitir la alimentación como práctica compleja, incidir superficialmente en ciertos factores estructurales, desatender las situaciones de precarización social y alimentaria y, como corolario, al relegar a un segundo plano la desigual distribución de la obesidad.

\section{METODOLOGÍA Y FUENTES DE DATOS}

A través de un estudio cualitativo, analizamos las medidas para hacer frente a la obesidad, adoptadas entre 2004-2019 por España, Argentina y Brasil. Como punto de partida, hemos tomado el año 2004, pues es cuando aparece la Global Strategy on Diet, Physical Activity and Health (DPAS) ${ }^{(18)}$ de la OMS. Del mismo modo, hemos considerado la Estrategia Regional y Plan de Acción para un enfoque integrado sobre la prevención y el control de las enfermedades crónicas, incluyendo el régimen alimentario, la actividad física y la salud de la Organización Panamericana de 
la Salud (OPS) ${ }^{(19)}$ que insta a todos los países miembros a desarrollar políticas sobre enfermedades crónicas no transmisibles (ECNT), entre ellas, la obesidad. Por tanto, y aunque el análisis ha incluido estos dos documentos y otros publicados por la OMS y sus oficinas regionales para Europa y América Latina recopilados de sus sitios web (un total de 17 distribuidos entre estrategias, planes, guías e informes), nos hemos centrado fundamentalmente en las medidas estatales de alcance nacional implementadas en el marco de la Estrategia NAOS (España) ${ }^{(20)}$, del Programa Nacional de Alimentación Saludable y Prevención de la Obesidad (Argentina) ${ }^{(21)}$ y de la Estrategia Intersectorial para el Control y la Prevención de la Obesidad (Brasil) ${ }^{(22)}$, además de documentos vinculados con las medidas mencionadas que cada uno de estos países ha venido implementando desde el sector salud.

Hemos recopilado y seleccionado intencionalmente estos textos mediante el acceso a los sitios web de las áreas ministeriales, teniendo en cuenta criterios de pertinencia teórica, clasificándolos por tema, año de publicación e implementación, por sus objetivos y las propuestas de acción. Hemos basado el análisis en un total de 46 documentos que cumplieron con los requisitos de inclusión (Tabla 1), distribuidos de la siguiente manera:
22 de España, 13 de Argentina y 11 de Brasil. Para analizarlos comparativamente hemos llevado a cabo un análisis temático ${ }^{(23)}$. Las categorías temáticas han sido: las definiciones sobre la obesidad; las evidencias disponibles; las causas identificadas; los costos asociados; los ámbitos de intervención; las soluciones promovidas y los objetivos perseguidos. Al finalizar, construimos matrices cualitativas con el análisis de los fragmentos incluidos en las categorías para establecer las comparaciones y la construcción del texto analítico.

Por último, y de forma complementaria al análisis pero como parte sustancial del argumento, hemos utilizado fuentes estadísticas para comprender la situación de precarización social y alimentaria y la prevalencia de obesidad de estas poblaciones durante este período. Dada la amplitud y diversidad de este tipo de fuentes en cada uno de estos países, solo hemos analizado los ítems de interés para el estudio. Para el caso de España, utilizamos encuestas sobre condiciones de vida, población activa y encuestas nacionales de salud; para Argentina, estadísticas sobre pobreza y tasa de desempleo, encuestas sobre inseguridad alimentaria y encuestas nacionales de factores de riesgo. Finalmente, para Brasil, la Pesquisa Nacional por Amostra de Domicílios, la Pesquisa de Orçamentos Familiares, la

Tabla 1. Criterios de inclusión y exclusión de los documentos recopilados.

\begin{tabular}{|c|c|}
\hline Criterios de inclusión & Criterios de exclusión \\
\hline $\begin{array}{l}\text { - Plan de Acción Mundial para la Prevención y el Con- } \\
\text { trol de Enfermedades no Transmisibles. } \\
\text { - Recomendaciones sobre dieta y actividad física. } \\
\text { - Estrategias globales y nacionales sobre dieta, acti- } \\
\text { vidad física y salud. } \\
\text { - Planes y programas de acción alimentaria y nutri- } \\
\text { cional de alcance nacional. } \\
\text { - Documentos de consenso sobre obesidad. } \\
\text { - Declaraciones de acción sobre prevención de la } \\
\text { - obesidad. } \\
\text { - Disposiciones, normativas, resoluciones y leyes re- } \\
\text { - Indicanadas con la prevención de la obesidad. } \\
\text { - Informes de monitoreo y evaluación. } \\
\text { - Programas, proyectos, materiales y guías destina- } \\
\text { dos a la población. }\end{array}$ & $\begin{array}{l}\text { - Plan de atención médica (general). } \\
\text { - Recomendaciones solo referidas a ingesta especí- } \\
\text { fica de alimentos / bebidas. } \\
\text { - Programas sobre actividad física o dieta para pobla- } \\
\text { ciones especíicas (campamento de verano, campa- } \\
\text { mento deportivo, hogares de ancianos, etc.) y/o de } \\
\text { alcance regional, provincial o municipal. } \\
\text { - Proyectos de investigación. } \\
\text { - Estrategias generales de promoción de la salud y } \\
\text { - prevención de las enfermedades. } \\
\text { - Materiales informativos (como folletería). } \\
\text { - Convenciones y premios. }\end{array}$ \\
\hline
\end{tabular}


Pesquisa Nacional de Saúde y la encuesta telefónica para la Vigilância de Fatores de Risco e Proteção para Doenças Crônicas.

Cabe señalar que el alcance de este estudio está limitado al análisis de documentos oficiales. Sin embargo, los hemos analizado como textos culturales cuyo acercamiento implicó examinar narrativas autorizadas e influyentes que construyen lo que es posible enunciar como la "realidad"(24).

\section{RESULTADOS}

\section{La obesidad como un problema global: causas universales, estrategias transnacionales}

Hace quince años, la OMS elaboró la DPAS, una herramienta integral para guiar a los Estados miembros en sus esfuerzos para prevenir las enfermedades crónicas no transmisibles $^{(15)}$. En ella, se consideró que la obesidad, además de impactar negativamente en la salud, sobrecargaba económicamente los sistemas sanitarios. Precisamente la necesidad de reducir este gasto impregnó el debate político, orientando las propuestas de este organismo hacia la promoción de la salud y la prevención del exceso de peso En la DPAS, la obesidad se concibió como una enfermedad epidémica vinculada con el aumento de comorbilidad y mortalidad, resultado de "dietas poco saludables y actividad física insuficiente" $y$, por tanto, de un "desequilibrio energético entre calorías consumidas y

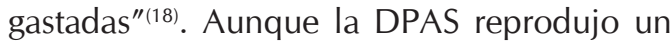
diagnóstico que identificaba factores ambientales, sociales e individuales como los principales causantes, subrayó que el crecimiento del suministro de energía alimentaria debido a la rápida transformación tecnológica y socioeconómica ocurrida en muchos países, incluidos los de bajos ingresos, estaba en la base del problema y, en consecuencia, propuso programas para evitarlo. Los fenómenos identificados como principales responsables fueron la mecanización del transporte y del trabajo, la comida procesada o las actividades de ocio pasivo, entre otros ${ }^{(25)}$. A una concepción cuasi aritmética de la obesidad, le correspondió un paquete de medidas que, aun siendo multifacéticas e intersectoriales, estuvieron destinadas a fomentar que las personas modificasen sus prácticas de consumo alimentario y los patrones de ejercicio y movilidad.

Sin embargo, en los últimos años, ha tenido que reorientar sus recomendaciones tras advertir que la obesidad ha seguido aumentando en los países que replicaron la DPAS a través de sus propias estrategias (Tabla 2).

A nivel discursivo, la OMS ha puesto énfasis en los factores microambientales, que configuran los patrones dietéticos (empleo, vivienda, desigualdades sociales) o en los factores macroambientales, que influyen en el consumo de alimentos (aumento de la oferta de alimentos, disminución de los precios de los alimentos, corporaciones alimentarias e industrialización de la agricultura) ${ }^{(26)}$. En esta línea, el Plan de Acción de Alimentación y Nutrición 2015-2020(27) va más allá de promover opciones saludables de alimentación y ejercicio físico, centrando el debate en la necesidad de mejorar los sistemas alimentarios. El plan reconoce que la obesidad es un problema que se asocia con la desigualdad y apoya el acceso universal a alimentos saludables, especialmente para los grupos más vulnerables. Al mismo tiempo, elabora orientaciones sobre cómo diseñar políticas fiscales sobre la dieta. No es casualidad que una parte de estos documentos fueran aprobados en 2013, a raíz de la Declaración de Viena sobre Nutrición y Enfermedades No Transmisibles en el Contexto de la Salud $2020^{(28)}$ coincidiendo con el impacto de la gran recesión económica en la salud de la población en situación de mayor precariedad social y alimentaria. Además, su contenido ha estado influido por la Declaración de Shanghái sobre Promoción de la Salud $^{(29)}$ en la que se establecen los compromisos adquiridos para que los entornos de consumo promuevan elecciones saludables mediante políticas de fijación de precios, para mejorar la salud pública y erradicar la pobreza, en el marco de los Objetivos de Desarrollo Sostenible (ODS). 
Tabla 2. Directrices internacionales relevantes en la prevención y control de la obesidad (2004-2019).

\begin{tabular}{|c|c|c|c|}
\hline Organismo & Año & Tipos de directrices & Descripción \\
\hline \multirow{6}{*}{$\begin{array}{l}\text { Organización } \\
\text { Mundial de la Salud }\end{array}$} & 2004 & Estrategia & Estrategia Mundial sobre Régimen Alimentario, Actividad Física y Salud \\
\hline & 2008 & Plan & $\begin{array}{l}\text { 2008-2013 Plan de Acción para la Estrategia Mundial para la Prevención y Control de } \\
\text { Enfermedades no Transmisibles }\end{array}$ \\
\hline & 2012 & Informe & Enfoques Poblaciones de la Prevención de la Obesidad Infantil \\
\hline & 2013 & Plan & $\begin{array}{l}\text { Plan de Acción Mundial para la Prevención y Control de las Enfermedades no Transmisibles } \\
(2013-2020)\end{array}$ \\
\hline & 2014 & Comisión & Comisión para acabar con la Obesidad Infantil. \\
\hline & 2016 & Informe & Políticas Fiscales para Régimen Alimentario y Prevención de Enfermedades No Transmisibles \\
\hline \multirow{5}{*}{$\begin{array}{l}\text { Organización } \\
\text { Mundial de la Salud } \\
\text { - Unión Europea }\end{array}$} & 2008 & Plan & Plan de Acción Europeo sobre Alimentación y Nutrición 2007-2012 \\
\hline & 2013 & Declaración & $\begin{array}{l}\text { Declaración de Viena sobre Nutrición y Enfermedades No Transmisibles en el Contexto de la } \\
\text { Salud } 2020\end{array}$ \\
\hline & 2014 & Guía & Obesidad y Desigualdades \\
\hline & 2015 & Plan & Plan de Acción Europeo sobre Alimentación y Nutrición 2015-2020 \\
\hline & 2019 & Informe & Informe sobre la Respuesta del Sistema de Salud a la Obesidad Infantil en la Región Europea. \\
\hline \multirow[t]{6}{*}{$\begin{array}{l}\text { Organización } \\
\text { Panamericana de } \\
\text { la Salud }\end{array}$} & 2007 & Estrategia & $\begin{array}{l}\text { Estrategia Regional y Plan de Acción para un Enfoque Integrado Sobre la Prevención y el } \\
\text { Control de Enfermedades Crónicas, incluyendo el régimen alimentario, la actividad física y } \\
\text { la salud. }\end{array}$ \\
\hline & 2012 & Estrategia & Estrategia para la prevención y el control de las enfermedades no transmisibles. \\
\hline & 2014 & Plan & $\begin{array}{l}\text { Plan de acción para la prevención y el control de las enfermedades no transmisibles en las } \\
\text { Américas 2013-2019. }\end{array}$ \\
\hline & 2014 & Plan & Plan de acción para la prevención de la obesidad en la niñez y la adolescencia. \\
\hline & 2015 & Informe & Alimentos y bebidas ultraprocesados en América Latina. \\
\hline & 2018 & Informe & $\begin{array}{l}\text { Políticas y programas alimentarios para prevenir el sobrepeso y la obesidad. Lecciones } \\
\text { aprendidas. }\end{array}$ \\
\hline
\end{tabular}

Fuente: Elaboración propia.

Por su parte, en el año 2007, la OPS diseñó la Estrategia Regional y el Plan de Acción para un Enfoque Integrado sobre la Prevención y el Control de Enfermedades Crónicas, donde proponía líneas de acción para su prevención y tratamiento. Sin embargo, ya en 2012, reconocía que, si bien se habían realizado grandes adelantos, no había logrado detenerse el avance de las enfermedades crónicas no transmisibles, proponiendo implementar acciones multisectoriales que consideren además los determinantes sociales de las enfermedades, como la obesidad. En el año 2014 afirmaba que

...los factores más importantes que promueven el aumento de peso y la obesidad [...] son el consumo elevado de productos de bajo valor nutricional y contenido alto de azúcar, grasa y sal [...]; la ingesta habitual de bebidas azucaradas y la actividad física insuficiente. Todos ellos son parte de un ambiente obesogénico. ${ }^{(27)}$
Instaba a los Estados miembros a implementar medidas dirigidas a "transformar el ambiente obesogénico actual en oportunidades para promover un consumo mayor de alimentos nutritivos y un aumento de la actividad física" ${ }^{\prime 27)}$. En línea con ello, las propuestas han estado asociadas con la promoción de la alimentación saludable, el mejoramiento del entorno escolar con respecto a la nutrición y la actividad física, políticas fiscales y reglamentación de la publicidad, promoción y etiquetado de alimentos, etc. Sin embargo, y también en línea con lo dicho en el caso de la OMS y los Objetivos de Desarrollo Sostenible, respecto al reconocimiento de la simultaneidad de situaciones de desnutrición y obesidad en contextos de pobreza y desigualdad, la mirada se ha ido centrando en los sistemas alimentarios buscando hacerlos sostenibles y sensibles a la nutrición, justos e inclusivos para la necesaria transformación de los ambientes obesogénicos en ambientes más saludables ${ }^{(30)}$. 


\section{Replicando diagnósticos, traduciendo políticas}

Del mismo modo que las recomendaciones de la OMS y la OPS, el punto de partida de las medidas implementadas en España, Argentina y Brasil (Tabla 3) es que la obesidad es una enfermedad epidémica, multicausal y costosa para el sistema de salud y la sociedad en su conjunto, pero que puede evitarse. Basándose en esta premisa, cada país ha diseñado su estrategia para hacerle frente, reproduciendo el

Tabla 3. Directrices y acciones relevantes en la prevención y control de la obesidad en España, Argentina y Brasil (2005-2019).

\begin{tabular}{|c|c|c|c|}
\hline País & Año & $\begin{array}{l}\text { Tipos de directrices y } \\
\text { acciones }\end{array}$ & Descripción \\
\hline \multirow[t]{11}{*}{ España } & 2005 & Estrategia & Estrategia para la Nutrición, la actividad física y la prevención de la obesidad (NAOS) \\
\hline & 2005 & Programa & Programa Piloto Escolar de Referencia para la Salud y el Ejercicio, contra la Obesidad (PERSE0). \\
\hline & 2005 & Normativa & $\begin{array}{l}\text { Código de co-regulación de la Publicidad de alimentos dirigida a menores, prevención de la } \\
\text { Obesidad y Salud (PAOS). }\end{array}$ \\
\hline & 2008 & Programa & $\begin{array}{l}\text { Programa que insta a los restaurantes a promover menúes saludables y equilibrados Proyecto } \\
\text { GUSTINO }\end{array}$ \\
\hline & 2011 & Ley & Ley de Seguridad Alimentaria y Nutrición 17/2011. \\
\hline & 2011 & Evaluación de programa & Evaluación y seguimiento de la estrategia NAOS: conjunto mínimo de indicadores. \\
\hline & 2013 & Programa & Programa para mejorar la salud global de la población adulta Fifty-fifty. \\
\hline & 2013 & Observatorio & Observatorio de la Nutrición y Estudio de la Obesidad. \\
\hline & 2014 & Plan & Plan de Acción Europeo sobre Obesidad Infantil, 2014-2020, JANPA (EU). \\
\hline & 2015 & Programa & $\begin{array}{l}\text { Programa de Alimentación, Nutrición y Gastronomía para Educación Infantil (PANGEI) "El gusto } \\
\text { es mío" }\end{array}$ \\
\hline & 2018 & Plan & $\begin{array}{l}\text { Plan de colaboración para la mejora de la composición de los alimentos y bebidas y otras } \\
\text { medidas. }\end{array}$ \\
\hline \multirow[t]{10}{*}{ Argentina } & 2008 & Ley & Ley 26396 de Trastornos Alimentarios. \\
\hline & 2009 & Estrategia & $\begin{array}{l}\text { Resolución 1083/09. Estrategia Nacional para la Prevención y Control de ENT y Plan Argentina } \\
\text { Saludable. }\end{array}$ \\
\hline & 2013 & Programa & Resolución 578/2013. Programa Nacional de Lucha contra el Sedentarismo. \\
\hline & 2014 & Ley & Iniciativa Argentina Libre de Grasas Trans (Industria Alimentaria). \\
\hline & 2014 & Guía & Guía Clínica sobre Diagnóstico y Tratamiento de Obesidad en Adultos. \\
\hline & 2015 & $\begin{array}{l}\text { Decreto/ } \\
\text { Proyecto }\end{array}$ & $\begin{array}{l}\text { Proyecto de Protección de la población vulnerable contra las enfermedades crónicas no } \\
\text { transmisibles. }\end{array}$ \\
\hline & 2016 & Guía & Guías Alimentarias para la Población Argentina (actualización). \\
\hline & 2016 & Programa & Resolución 732/16. Programa de Alimentación Saludable y Prevención de la Obesidad. \\
\hline & 2018 & Mesa de discusión & $\begin{array}{l}\text { Mesa de Discusión sobre diferentes opciones de etiquetado frontal entre la Secretaria de } \\
\text { Gobierno de Salud y el Ministerio de Producción y Trabajo. }\end{array}$ \\
\hline & 2019 & Plan & Plan Nacional de Prevención de Sobrepeso y Obesidad en Niños, Niñas y Adolescentes \\
\hline \multirow[t]{8}{*}{ Brasil } & 2006 & Manual & Obesidade. Cadernos de Atenção Básica, 12 Série A. Normas e Manuais Técnicos \\
\hline & 2011 & Plan & $\begin{array}{l}\text { Plano de ações estratégicas para o enfrentamento das doenças crônicas não transmissíveis } \\
\text { (DCNT) no Brasil. }\end{array}$ \\
\hline & 2012 & Política & Política Nacional de Alimentação e Nutrição \\
\hline & 2013 & Ley & $\begin{array}{l}\text { Portaria 424/GM/MS de 2013. Redefine as diretrizes para a organização da prevenção e do } \\
\text { tratamento do sobrepeso e obesidade como linha de cuidado prioritária da Rede de Atenção à } \\
\text { Saúde das Pessoas com Doenças Crônicas. }\end{array}$ \\
\hline & 2104 & Manual & Obesidade. Cadernos de Atenção Básica 38. \\
\hline & 2014 & Guía & Guia alimentar para a população brasileira (revisão). \\
\hline & 2014 & Estrategia & $\begin{array}{l}\text { Estratégia Intersetorial de Prevenção e Controle da Obesidade: recomendações para estados e } \\
\text { municípios. }\end{array}$ \\
\hline & 2015 & Marco de referencia & Marco de referência da vigilância alimentar e nutricional na atenção básica. \\
\hline
\end{tabular}


diagnóstico sobre sus causas y replicando algunas de las medidas propuestas a nivel global y regional.

Preocupada por el aumento de las enfermedades crónicas no transmisibles y por la vinculación de la obesidad en dicho aumento, España responde con prontitud (en 2005) al mandato de la OMS a través de la estrategia NAOS, a partir de la cual se subraya que "el conocimiento exhaustivo de las causas y de sus múltiples y complejas interrelaciones es esencial para cambiar los hábitos públicos e intervenir en las causas de la obesidad"(20). Estas se concretan básicamente en dos: a) el aumento sostenido del sedentarismo, y b) el cambio en los patrones de alimentación durante los últimos 40 años que han ido de más a menos saludables ${ }^{(31)}$. Los ambientes obesogénicos han sido considerados los culpables de estas tendencias ${ }^{(20)} y$ ha llevado a una serie de propuestas que "fomentan un cambio decisivo y sostenido hacia una dieta más saludable y actividad física re-

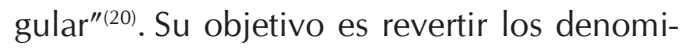
nados "estilos de vida no saludables" a través de acciones multifacéticas, proponiendo programas que requieren la colaboración de numerosos actores sociales e intervenciones en diferentes ámbitos (escolar, laboral, sanitario, comunitario) y que se han visto impulsados por la Ley 17/2011 de Seguridad Alimentaria y Nutrición. Se creó también un observatorio para estudiar la evolución de la obesidad; se introdujo más información nutricional en los envases de alimentos; se establecieron algunos acuerdos con los restaurantes y las industrias para ofrecer menús saludables; se prohibió la venta de alimentos procesados en las escuelas; y también se capacitó a los equipos del primer nivel de atención ${ }^{(15)}$.

Si bien las medidas Ilevadas a cabo han tratado de involucrar a distintos actores sociales, cuando se analizan en su conjunto, es posible ver que hay constreñimientos específicos para promover el sugerido decisivo y sostenido cambio. Por ejemplo, la industria española de alimentos y bebidas considera que es más efectivo mejorar la información en el etiquetado de productos y en la publicidad y promover estilos de vida saludables y consumo responsable en lugar de penalizar los alimentos procesados y los refrescos con impuestos adicionales:

Sería un error considerar que los esfuerzos en innovación y reformulación son la solución a los problemas relacionados con enfermedades como la obesidad. Aparte de la dieta, es imprescindible considerar todos los factores que pueden tener impacto en la salud: predisposición genética, estilos de vida, niveles de actividad física y factores socioeconómicos. ${ }^{(15)}$

Aunque las empresas siguen trabajando voluntariamente en la reformulación de los productos, reduciendo la sal, los azúcares y las grasas saturadas, insisten en educar a los consumidores para que opten por una dieta equilibrada y practiquen actividad física.

Si bien el gobierno ha optado por no gravar los Ilamados alimentos no saludables, en un intento tímido de subsidiar los productos más saludables ha llevado a implementar el menú de frutas escolares, una iniciativa promovida por la Comisión Europea cuyo objetivo es financiar la distribución gratuita de algunas frutas y verduras para niños de 6 a 12 años en las escuelas participantes. Por otro lado, si bien se afirma que las iniciativas desarrolladas priorizan a los niños, los jóvenes y los grupos de población más desfavorecidos, con enfoque de género, evitando las desigualdades en salud centrándose en los grupos específicos afectados ${ }^{(20)}$, las campañas para prevenir la obesidad se han dirigido principalmente al público en general y, en particular, a niños y adolescentes, buscando convencerlos de los beneficios positivos que obtendrán mediante la adopción de un estilo de vida saludable. Con ese fin se han diseñado guías, talleres, juegos, spots audiovisuales, etc., que se centran en cambiar el comportamiento de las personas diciéndoles qué, cuándo, dónde y cuánto comer o hacer ejercicio. El lema de la NAOS es "Come sano y muévete". Los destinatarios de estos mensajes son considerados meros receptores de las recomendaciones propuestas por el conocimiento experto (al que se han ido sumando 
los mensajes de deportistas o cocineros famosos) que refuerzan los discursos sobre prácticas alimentarias apropiadas e inapropiadas. Recientemente, se han añadido otras iniciativas dirigidas a niños entre 3 y 6 años con un enfoque gastronómico para inculcar la dieta mediterránea y "mejorar su cultura alimentaria". El programa "El gusto es mío" pretende -además de inculcar hábitos saludables que los niños aprendan en la escuela- a apreciar sabores, olores y texturas ${ }^{(32)}$. En el ámbito laboral y doméstico, por su parte, se han hecho campañas "para ser más activos", promoviendo "substituir las pausas de café por pausas para caminar", "hacer reuniones de trabajo caminando", "no estar más de dos horas seguidas sentados" o "romper con el tiempo sentado cada 30 minutos"(33).

Por su parte, Argentina, a diferencia de España, diseña su estrategia integral años más tarde, dado que la atención sanitaria estaba centrada en atender las problemáticas vinculadas con el hambre y la desnutrición en el contexto de la crisis de 2001. Con todo, la obesidad ya había ingresado en la agenda sanitaria a partir de diferentes relevamientos epidemiológicos que fueron justificando su construcción como objeto de política de salud a partir del período 2003-2005. En 2008, se promulga la Ley 26396 de Trastornos Alimen$\operatorname{tarios}^{(34)}$ que insta a crear el Programa Nacional de Prevención y Control de los Trastornos Alimentarios, incluyendo la obesidad junto con la bulimia y la anorexia. No es hasta la Resolución 1083/2009 cuando se reubica a la obesidad dentro de una Estrategia Nacional para la Prevención y Control de Enfermedades Crónicas y del Plan Nacional Argentina Saludable a instancias de las recomendaciones de la OPS. Hasta ese momento, la obesidad no era considerada un problema que requiriera medidas particulares para su prevención o atención, más allá de la iniciativa de "Argentina Libre de Grasas Trans" ${ }^{\prime \prime 35)}$ que, por ley, a diferencia de España, estipula la restricción del contenido de los ácidos grasos trans en la producción industrial. Finalmente, en 2016, se comienza a implementar el Programa Nacional de Alimentación Saludable y Prevención de la Obesidad (PNASPO $)^{(35)}$. Al igual que la estrategia
NAOS en España, está en concordancia con las directrices de la OMS y con la Estrategia Regional y Plan de Acción para un enfoque integrado sobre la prevención y control de las enfermedades crónicas no transmisibles, de la OPS, de 2007 y de 2014. También parte de un diagnóstico y del impacto económico de la obesidad, afirmando que:
...el sobrepeso y la obesidad, [están] relacionados con la alimentación no saludable y el sedentarismo [...] y los costos directos para el sistema de salud e indirectos para la sociedad son elevados [...]. La obesidad se asocia a enfermeda- des crónicas y mayor uso de recursos del sistema de salud y fuera de él. ${ }^{(35)}$

Su propósito es ofrecer un enfoque integral de la alimentación saludable y del problema de la obesidad, que abarque los aspectos que determinan su incremento en el marco de un ambiente obesogénico, y que lo identifique como el responsable de la tendencia. Según el programa, la elevada oferta de productos alimentarios con alto contenido de sal, grasas saturadas y azúcares libres, la existencia de publicidad inadecuada, la realización de dietas inadecuadas y la escasa realización de actividad física son la expresión de este ambiente. Si bien -al igual que la NAOS- propone líneas de acción variadas (promoción y la regulación de la oferta de alimentos a través de acuerdos con la industria -para la reducción de la sal, grasas trans y azúcares-y con otros actores y propuestas de cambios en la legislación sobre el etiquetado frontal de alimentos y sobre la publicitad alimentaria, se plantea una intervención sobre el comportamiento individual a través de la promoción de la dieta saludable y la actividad física en diferentes ámbitos como los domésticos, laborales o escolares. Para ello se diseñan, también como en el caso de España, materiales gráficos, audiovisuales y guías alimentarias que difunden mensajes como "Comé rico, comé saludable"; "Tu pausa activa, sumá 30 minutos de actividad física todos los días"; "Aumentá el consumo de frutas, verduras y de pescado; "Disminuí el consumo 
de alimentos ultraprocesados", en los que se recomienda a toda la población adoptar comportamientos saludables. $\mathrm{Al}$ igual que en España, se ha añadido otra iniciativa destinada a la población infantil: el Plan Nacional de Prevención de Sobrepeso y Obesidad en Niños, Niñas y Adolescentes ${ }^{(36)}$, en el que se entiende a la "obesidad como expresión de la malnutrición" en el marco de los acuerdos con la OPS y los Objetivos de Desarrollo Sostenible y, por lo tanto, se busca atender "a los que más sufren la malnutrición en todas sus formas" bajo el enfoque de derechos y de género. Si bien a nivel declarativo busca el fortalecimiento de la educación alimentaria y la actividad física, la sensibilización de la comunidad, el fortalecimiento de la calidad nutricional de los programas alimentarios, la promoción de sistemas alimentarios sostenibles y la regulación de los entornos y productos alimenticios ${ }^{(36)}$, ha tenido como actividades centrales la información y la capacitación de docentes y cocineros de comedores escolares, además de la publicación de guías, y manuales de capacitación y materiales lúdico-educativos destinados a la población infantil con mensajes como "Comé en colores", "Comé frutas y verduras variadas", "Activá tu cuerpo todos los días", entre otros.

Finalmente, en Brasil, las directrices para organizar acciones de prevención y tratamiento de la obesidad datan de fines de la década de 1990 con la definición de la Política Nacional de Alimentación y Nutrición (PNAN), revisada en $2012^{(37)}$. Con una historia de hambre, desnutrición y otras deficiencias nutricionales, la población brasileña ha experimentado transformaciones sociales que resultaron en una reducción de la pobreza y la exclusión social y, en consecuencia, el hambre y la desnutrición. Por otro lado, ha habido un aumento del sobrepeso en todos los sectores de la población, lo que indica un nuevo escenario. A pesar de los diferentes contextos socioculturales y económicos, como los de España, el PNAN atribuye las causas de la obesidad a la disminución de la actividad física y los patrones de alimentación poco saludables y también propone acciones en varios sectores "desde la producción hasta la comercialización final de alimentos y garantizar entornos que permiten el cambio de conducta de los individuos y la sociedad"(37). Los problemas de la obesidad se vinculan con los sistemas alimentarios, incorporando al debate la cuestión de la soberanía alimentaria.

Al mismo tiempo, se atribuye al individuo la responsabilidad para la elección de alimentos y, así, la necesidad de "invertir en herramientas y estrategias de comunicación y educación en salud que apoyen a los profesionales de la salud"(37) en la difusión de información alimentaria y nutricional. Al igual que en Argentina y España, las medidas se basan en un enfoque individual a través de la promoción de una dieta saludable y actividad física teniendo en cuenta el ciclo vital con mensajes como "Practique al menos 30 minutos de actividad física todos los días", "Mantenga el peso dentro de los límites saludables" ${ }^{\prime(38)}$, y pautas sobre compras y cuidado de la higiene y almacenamiento de alimentos. La discusión de los límites que enfrentan los propios individuos para modificar sus "elecciones" personales es frágil y se fundamentan en la disponibilidad de alimentos saludables en el entorno, como la escuela y el trabajo. Pero en 2014, a través de la Cámara Interministerial de Seguridad Alimentaria y Nutricional, se formula el Plan Intersectorial para Combatir la Obesidad, diseñándose, de manera singular, una estrategia intersectorial para estados y municipios desarrollada por diversos ministerios (salud, agricultura, educación, trabajo, medio ambiente, entre otros), la sociedad civil y miembros de la OPS/OMS.

Esta articulación se justifica por la necesidad de actuar conjuntamente entre gobierno y representantes de movimientos y organizaciones con el objetivo de velar por el cumplimiento del derecho a la alimentación ${ }^{(39)}$. Uno de los objetivos de la estrategia es "promover la alimentación adecuada y saludable y la actividad física en el ambiente que vivimos"(22). Como en los otros países, los denominados factores obesogénicos son considerados los responsables de esta tendencia, focalizando nuevamente sus acciones también en el cambio de comportamientos individuales y, sobre todo, a través de la reducción del 
consumo de productos ultraprocesados. Las acciones de educación y comunicación estimulan la autovigilancia y las prácticas de educación alimentaria y nutricional con individuos y grupos de población para que opten por alimentos saludables. Además, tanto las guías alimentarias como otros materiales didácticos divulgan mensajes para favorecer cambios en la dieta y en la actividad física como "Haga que los alimentos frescos o mínimamente procesados sean la base de su dieta", "Evite los alimentos ultraprocesados" y "Si tiene habilidades culinarias, intente desarrollarlas y compartirlas, especialmente con niños y jóvenes".

Sin embargo, a diferencia de España y Argentina, en Brasil, el primer eje de acción de su estrategia da prioridad a "facilitar el acceso físico de las comunidades y familias a los alimentos y preparaciones tradicionales $y$, por otro lado, ampliar la oferta de alimentación adecuada y saludable en los equipamientos públicos del país"(22). Así, en el marco de esta política, se proponen medidas integrales que pretenden también fomentar la agricultura familiar, valorizar el consumo de alimentos regionales y preparaciones tradicionales, promover la sustitución de alimentos ultraprocesados y procesados por frutas y hortalizas, granos integrales, leguminosas, oleaginosas y pescados, fomentar la comercialización en circuitos más pequeños o la tributación de alimentos e insumos. Hacer compras institucionales de alimentos producidos por granjas familiares a través de convocatorias públicas en instituciones filantrópicas e instalaciones públicas y alentar el consumo de pescado en las comidas escolares son ejemplos de algunas de las acciones propuestas ${ }^{(22)}$. Finalmente, aquí también se ha establecido un acuerdo de compromiso entre el Ministerio de Salud y asociaciones de la industria alimentaria para reducir las cantidades de azúcar, grasa y sodio en los alimentos.

\section{Obesidad en contextos precarios}

En los tres países, la mayor parte de las acciones recalcan los beneficios de seguir estilos de vida saludables y se han dirigido, sobre todo, a la población en general (o a ciertos grupos etarios) con mensajes y medidas similares entre sí. Apenas hemos podido encontrar programas destinados a los grupos de población más desfavorecidos o con enfoque de género. Sin embargo, la información epidemiológica disponible en cada uno de los países permite afirmar que la obesidad no ha cesado de aumentar y que afecta principalmente a sectores de ingresos y/o nivel instrucción más bajos y, dentro de las poblaciones en situación de precarización social y alimentaria, las mujeres, en algunos casos, son las más afectadas.

En España, se ha producido un aumento creciente del exceso de peso. Según la Encuesta Nacional de Salud (ENS), la obesidad ha pasado del $15,5 \%$ en $2006^{(40)}$ a $17,45 \%$ en $2017^{(41)}$. Los datos evidencian que ha aumentado en ambos sexos, que se incrementa con la edad, y que es notablemente superior entre las personas con niveles de ingresos e instrucción más bajos, especialmente las mujeres. La prevalencia alcanza el $28,27 \%$ entre las personas sin estudios reglados, mientras que se sitúa en el 9,58\% entre los universitarios. Por su parte, esta diferencia es aún mayor entre las mujeres sin estudios, alcanzando el 30,25\%. Un dato epidemiológico relevante es que la prevalencia ha aumentado más rápido entre los grupos socialmente más desfavorecidos. Mientras en los sectores de ingresos altos la obesidad incluso ha decrecido un punto entre 2006 y 2017, situándose en el 9,29\%, en el mismo periodo aumentó 3 puntos entre los sectores de bajos ingresos, elevándose a $22,37 \%$, más del doble. Si observamos las diferencias de género, la obesidad se multiplica por más de 3 en el caso de las mujeres más pobres $(23,98 \%)$ frente al $7,26 \%$ del grupo de más altos ingresos (Tabla 4). Si anclamos estos datos con el contexto social y económico español, podremos ir comprendiendo por qué la obesidad afecta estos sectores.

En este sentido, en el contexto europeo, España se destaca por ser uno de los países en los que más ha crecido la desigualdad social en la última década y se ha precarizado el trabajo. Desde el comienzo de la crisis 
Tabla 4. Prevalencia (\%) de sobrepeso y obesidad. Población, fuente específica de estudio y año en España, Argentina y Brasil.

\begin{tabular}{|c|c|c|c|c|c|c|}
\hline País & Fuente & Año o período & $\begin{array}{l}\text { Población } \\
\text { de estudio }\end{array}$ & $\begin{array}{l}\text { Exceso de } \\
\text { peso }^{1}(\%)\end{array}$ & $\begin{array}{c}\text { Sobrepeso } \\
(\%)\end{array}$ & $\begin{array}{l}\text { Obesidad } \\
\text { (\%) }\end{array}$ \\
\hline \multirow[t]{3}{*}{ España } & \multirow[t]{3}{*}{$\operatorname{ENSE}^{(40,41,42)}$} & 2006 & \multirow[t]{3}{*}{ Mayor de 18 años } & 48,7 & 33,2 & 15,5 \\
\hline & & $2011 / 2012$ & & 53,7 & 36,7 & 17,0 \\
\hline & & $2017 / 2018$ & & 54,4 & 36,9 & 17,5 \\
\hline \multirow[t]{4}{*}{ Argentina } & \multirow[t]{4}{*}{$\operatorname{ENFR}^{(50,51,1,2,53)}$} & 2005 & \multirow[t]{4}{*}{ Mayor de 18 años } & 49,0 & 34,4 & 14,6 \\
\hline & & 2009 & & 53,4 & 35,4 & 18,0 \\
\hline & & 2013 & & 57,9 & 37,1 & 20,8 \\
\hline & & 2018 & & 61,6 & 36,3 & 25,3 \\
\hline \multirow[t]{4}{*}{ Brasil } & $\mathrm{POF}^{(58,59)}$ & $2002 / 2003$ & \multirow[t]{2}{*}{20 años o más } & 40,6 & 29,5 & 11,1 \\
\hline & & $2008 / 2009$ & & 49,0 & 34,2 & 14,8 \\
\hline & PNS(60) & 2013 & \multirow[t]{2}{*}{18 años 0 más } & 56,9 & 36,1 & 20,8 \\
\hline & VIGITEL ${ }^{(61)}$ & 2018 & & 55,7 & 35,9 & 19,8 \\
\hline
\end{tabular}

Fuente: Elaboración propia basada en datos disponibles.

${ }^{1}$ Exceso de pes $0=$ sobrepes $0+$ obesidad.

ENSE = Encuesta Nacional de Salud de España; ENFR= Encuesta Nacional de Factores de Riesgo; POF= Pesquisa de Orçamentos

Familiares; PNS= Pesquisa Nacional de Saúde; VIGITEL= Vigilância de fatores de risco e proteção para doenças crônicas por inquérito telefônico.

económica mundial, en 2008, el gobierno respondió a los efectos iniciales de la recesión económica centrando sus esfuerzos en los rescates bancarios, desregulando las relaciones laborales, reduciendo el gasto en salud y aumentando los impuestos directos $\mathrm{e}$ indirectos ${ }^{(43)}$. Aunque algunos indicadores macroeconómicos han mejorado desde 2015 y según la Encuesta de Población Activa $^{(44)}$ la tasa de desempleo cayó al 16,55\% en el cuarto trimestre de 2017, el número de desempleados aún es de casi 3,5 millones de personas. Además, la calidad de los trabajos ha empeorado, con contratos temporales y salarios bajos que no permiten que trabajadores salgan de la pobreza ${ }^{(45)}$. Tanto la pobreza como la desigualdad de ingresos se encuentran entre las más altas de la Unión Europea ${ }^{(46)}$. Según el índice AROPE, la proporción de la población española en riesgo de exclusión social aumentó del 23,3\%, en 2007, al $26,1 \%$, en 2018 , y actualmente afecta a 12,3 millones de personas ${ }^{(47)}$, muchas de las cuales dependen de la asistencia social ${ }^{(48)}$. Este creciente empobrecimiento ha tenido consecuencias en varias áreas de la vida diaria para los grupos más vulnerables, particularmente, en su alimentación. Sin embargo, las consecuencias socioeconómicas y sanitarias de la precarización no se conocen bien porque durante muchos años la investigación sobre el acceso de las personas a los alimentos ha sido esporádica y fragmentada ${ }^{(49)}$. Por tanto, poco se sabe sobre el grado de inseguridad alimentaria en España.

En Argentina también sucede algo similar. La Encuesta Nacional de Factores de Riesgo (ENFR), de 2018 ${ }^{(50)}$, muestra que el exceso de peso ha aumentado en todos los grupos etarios, en ambos sexos y en todos los sectores socioeconómicos. En el caso de la obesidad, ha aumentado desde el 2005, alcanzando en 2018 el $25,3 \%$. Su frecuencia fue significativamente mayor en el grupo con menor nivel de instrucción respecto del de mayor nivel de instrucción. Asimismo, fue más alta en los grupos de más bajos ingresos $(27,6 \%)$ con respecto a los grupos de mayores ingresos $(19,6 \%)$. Por tanto, en la obesidad hay un claro gradiente descendente por nivel educativo y socioeconómico, siendo las poblaciones en situación de vulnerabilidad las que padecen más obesidad. Si se tiene en cuenta su progresión, en los grupos de menores ingresos la obesidad ha aumentado en mayor proporción: mientras en 2005, los grupos de menores ingresos alcanzaban el $16,3 \%$ y los de mayores ingresos el $13,1 \%$, en 2018, Ilegaron 
al $27,6 \%$ y al $19,6 \%$, respectivamente (Tabla 4). Respecto de los indicadores socioeconómicos, luego de la recuperación de la crisis del año 2001, la situación empeoró nuevamente con la crisis financiera internacional de 2008, instalándose a partir de 2015 un esquema neoliberal para sortear problemas de la economía local, regional e internacional. Con políticas de ajuste fiscal y monetario, la inflación se extendió $(52,1 \%$ de variaciones interanual de precios de alimentos en 2019) (54) y hasta 2019 no fue acompañada por un reacomodamiento de los ingresos, afectando no solo el poder adquisitivo sino acrecentando la desigualdad social. Así, el porcentaje de personas en la pobreza pasó del 31\% en 2006 (luego de un pico del $50 \%$ en la crisis de 2002) a un $35,4 \%$ en el 2019, y la tasa de desempleo pasó del 8,7\% en 2006 al $10,6 \%$ en $2019^{(55)}$. Esto afectó profundamente el acceso a los alimentos de los sectores más desfavorecidos, con cálculos que afirman que la inseguridad alimentaria (la dificultad de acceder a los alimentos en cantidad y calidad por motivos económicos) ha afectado en 2019 al 22,2\% de la población urbana y, dentro de ella, la experiencia de sufrir hambre al 9,3\%(56), confirmando para el contexto argentino lo analizado en sucesivas investigaciones por Aguirre ${ }^{(57)}$ en relación con la obesidad en situaciones de pobreza.

Finalmente, en Brasil, según los datos de la Pesquisa de Orçamentos Familiares (POF), la prevalencia de exceso de peso en adultos ha pasado del $40,6 \%$ en $2002-2003^{(58)}$ al $49,0 \%$ en $2008-2009^{(59)}$. La distribución económica de la obesidad se acerca a la distribución observada para el exceso de peso. En las mujeres, la mayor incidencia de exceso de peso se encuentra en las clases de ingresos medios. En los hombres, las frecuencias de exceso de peso aumentaron con los ingresos. Por su parte, la Pesquisa Nacional de Saúde (PNS) de $2013^{(60)}$ señaló el aumento continuo del sobrepeso y la obesidad $(36,1 \%$ y $20,8 \%$, respectivamente). Finalmente, los datos de VIGITEL de $2018^{(61)}$ mostraron el aumento continuo en la frecuencia de sobrepeso $(35,9 \%)$ y obesidad $(19,8 \%)$ en relación con la POF 2002-2003 y 2008-2009 y una tendencia a la baja en relación con la PNS 2013. Los datos de VIGITEL 2018 también revelan que entre los hombres, la frecuencia de sobrepeso fue mayor en los estratos extremos del nivel de instrucción y entre las mujeres disminuyó notablemente con el aumento del nivel de instrucción. Sin embargo, la frecuencia de obesidad en ambos sexos disminuyó con el aumento del nivel de instrucción, especialmente para las mujeres (Tabla 4).

En relación con la precarización social y económica en Brasil, la pobreza ha sido una parte constitutiva de su historia, derivada de la desigualdad social, producto de políticas que han privilegiado la concentración de ingresos durante décadas. Pero como resultado de las inversiones en políticas destinadas a superar la pobreza y la desnutrición, hubo un período de marcada reducción de la desigualdad, entre 2001 y 2014, que no fue acompañada con una disminución de la obesidad. No obstante, a finales de 2014 ha comenzado un período crítico debido al desempleo con la caída del ingreso promedio, aumento de la desigualdad y, en consecuencia, aumento de la pobreza. Desde finales de 2014 hasta finales de 2017, el aumento de la pobreza fue del $8,38 \%$ al $11,18 \%{ }^{(62)}$. Este aumento se combina con la falta de redes de seguridad social, acentuada por una serie de políticas de reducción del gasto público que han congelado las inversiones en áreas clave como salud, educación, trabajo y seguridad social y, más directamente, en el área de seguridad alimentaria y nutricional, la extinción del Consejo Nacional de Seguridad Alimentaria y Nutricional. Hasta 2014, las políticas eliminaron a Brasil del Mapa del Hambre, pero en la actualidad van en sentido contrario.

En estos tres países, como una de las herencias de las políticas de austeridad fiscal y monetaria implementadas en los últimos años, la obesidad constituye un epítome de la precarización, cristalizando una forma particular de "cuerpos precarios", los cuales encarnan las desigualdades sociales. 


\section{La desatención de los determinantes sociales en las estrategias contra la obesidad}

Esta distribución desigual de la obesidad en contextos precarios ha sido contemplada en las estrategias analizadas de forma modesta. Ello contrasta, como hemos afirmado, con el hecho de que las medidas principales reconocen la incidencia de las condiciones económicas, físicas y sociales en relación con la obesidad. La Ley de Seguridad Alimentaria y Nutrición del año 2011, aprobada en el punto álgido de la crisis en España, señala que la obesidad afecta, sobre todo, a las clases más desfavorecidas socialmente e indica a la estrategia NAOS priorizar las medidas dirigidas a esos grupos ${ }^{(63)}$. Ese mismo año se publicó la Evaluación y Monitoreo de la Estrategia $\mathrm{NAOS}^{(64)}$, una herramienta específica para la evaluación de todas las acciones realizadas utilizando, entre otros, indicadores de género y clase social y, en 2013, se estableció el Centro de Observación para la Nutrición y el Estudio de la Obesidad, entre cuyas funciones se encuentra detectar cambios en la prevalencia de la obesidad en relación con las desigualdades sociales y los factores ambientales ${ }^{(15)}$.

Aunque desde 2015, y tras la reorientación de las políticas públicas derivadas del Plan de Acción Europeo de Alimentación y Nutrición 2015-2020(27), algunos programas han dirigido sus acciones a personas de estratos socioeconómicos más bajos, y han adoptado enfoques similares vinculados con el cambio de comportamiento. Un ejemplo es el proyecto POIBA (2010-14) ideado por la Agencia de Salud Pública de Barcelona para niños de 11 a 12 años, la mitad de los cuales vive en los barrios más pobres de la ciudad $^{(65)}$. Los objetivos de este programa son promover la actividad física y la alimentación saludable a través de talleres educativos y actividades recreativas que involucren a maestros, niños y sus familias. Una evaluación inicial de la eficacia del programa reveló una disminución en la tasa de obesidad a corto plazo. Sin embargo, estos fueron mayores y más duraderos entre los niños de las zonas más acomodadas ${ }^{(66)}$.
También sucede algo similar en Argentina. A pesar del reconocimiento del aumento de la prevalencia de la obesidad en toda la población, especialmente en los sectores más desfavorecidos y de la incidencia de los entornos, las medidas tienden a obviar la distribución social de la obesidad. Aunque en el año 2015 comenzó a implementarse el Proyecto de Protección de la Población Vulnerable contra las ECNT ${ }^{(67)}$, sus acciones se han centrado nuevamente en la promoción de actividad física y de alimentación saludable enfocadas en cambiar comportamientos, aun en las poblaciones desfavorecidas socialmente. Esto se ve reforzado en los mismos mensajes: en un material audiovisual del ministerio de salud se reconoce que comer saludable depende de múltiples factores, entre ellos, el precio de los alimentos, pero también se afirma que "dentro de cada presupuesto uno puede mejorar la alimentación" ${ }^{\prime \prime 68)}$. Otra recomendación sugiere a la población: "preferí la alimentación casera, es más sana y económica".

En Brasil, las políticas específicas para abordar la obesidad también se han centrado en medidas que toman poco en cuenta la desigual distribución de la obesidad, a pesar de que las políticas de Seguridad Alimentaria y Nutricional han enfatizado el acceso y la producción de alimentos y la valorización de la agricultura familiar entre grupos más vulnerables. Dentro del alcance del Programa Nacional de Alimentación Escolar, la aplicación de al menos el 30\% de los recursos para la adquisición de estos alimentos es uno de los mayores avances en términos de establecer criterios que impacten socialmente. No obstante, esta articulación todavía se presenta como un gran desafío, pues introducir productos de pequeños campesinos en las comidas escolares no es una acción trivial tanto para compradores como para vendedores ${ }^{(69)}$, aún más teniendo en cuenta los efectos de las actuales políticas de austeridad y el desmantelamiento de los programas previos impuestos por el actual gobierno. Por otro lado, la Guía Alimentaria para la Población Brasileña $^{(70)}$ de 2014, que en muchos aspectos ha sido innovadora en su concepción de 
la alimentación como práctica social compleja, señala que la información es útil para aquellos que sufren enfermedades específicas, pero sin distinción de clase social en las recomendaciones pues, como todas las guías, están dirigidas a la población general. Con todo, amplía el debate más allá del sector de la salud y enfatiza el concepto de "comida de verdad", que se basa en alimentos frescos o mínimamente procesados y preparaciones culinarias, como criterio de clasificación de los alimentos. En este punto, introduce un fuerte elemento de confrontación con la industria alimentaria aunque al mismo tiempo le atribuye al individuo la responsabilidad de "eliminar obstáculos".

$\mathrm{Si}$, como afirmamos, desde los organismos internacionales se reconocen "las interacciones complejas entre las opciones personales, las normas sociales y los factores económicos y ambientales"(18) y la necesidad de tener en cuenta "Ias circunstancias culturales y socioeconómicas locales" y "los determinantes sociales para reducir la desigualdad en la distribución de la obesidad"(27), ¿ por qué no se han llevado a cabo estudios centrados en examinar la naturaleza de dichas interacciones, explorando las manifestaciones locales de esta problemática y, sobre esta base, plantear propuestas para transformar los contextos adversos y los determinantes sociales que condicionan la obesidad? Algunas iniciativas pueden tomarse como ejemplo. Es el caso de los estudios desarrollados en Brasil sobre el entorno alimentario desde la perspectiva del acceso a alimentos saludables (especialmente frutas y verduras), los cuales están mapeando lugares y relacionándolos con el nivel de ingresos a fin de incidir en las políticas públicas ${ }^{(71,72,73)}$. Destacamos también los estudios realizados por el Núcleo de Pesquisas Epidemiológicas em Nutrição e Saúde de la Universidade de São Paulo sobre las políticas alimentarias y nutricionales y los Conselhos Municipais de Segurança Alimentar e Nutricional, que asumen que una dieta saludable implica la transformación del sistema alimentario. En Argentina, se puede citar una investigación realizada sobre entornos obesogénicos en situaciones de vulnerabilidad social en $2019^{(74)}$. En el caso de España, se desconocen iniciativas sobre estudios para la modificación de los entornos en las áreas socialmente más desfavorecidas. Como mucho, se señalan, al igual que en Argentina, las acciones que supuestamente potenciarían entornos alimentarios saludables. Por ejemplo, una mayor regulación publicitaria y del etiquetado, impuestos o subvenciones a los alimentos y reformulación alimentaria de obligado cumplimiento.

\section{DISCUSIÓN Y CONCLUSIONES}

En un contexto epidemiológicamente similar en ciertos aspectos, pero cultural, político y económico distinto, España, Argentina y Brasil han sido activos en el establecimiento de medidas para hacer frente a la obesidad y lo han hecho siguiendo, en parte, las directrices promovidas por la OMS, la UE y la OPS. Aun así, ninguno ha conseguido revertir la tendencia. Sostenemos que las razones hay que buscarlas en el excesivo énfasis puesto en la responsabilidad individual en contextos de precarización social y alimentaria. Porque aunque la tipología de las medidas propuestas en los tres países ha sido variada, ha sido menos plural en cuanto a su naturaleza y alcance. La mayoría de los programas se centran en promocionar un modelo estandarizado de dieta y ejercicio físico y, por lo tanto, de autovigilancia sobre las personas, indicando qué y cuánto deben comer o moverse ${ }^{(2)}$. Los intentos por mejorar los estilos de vida adoptan la forma de acciones destinadas a educar al público (especialmente a los niños) pero a menudo no tienen en cuenta la naturaleza social de la alimentación y la cultura ${ }^{(2,15)}$. Como hemos visto, aunque el exceso de peso afecta a quienes tienen menos ingresos, nivel de instrucción $y$, entre estos, a las mujeres, y durante estos años ha aumentado con mayor rapidez o en mayor proporción entre estos grupos sociales, las recomendaciones sobre los patrones "ideales" para comer, cocinar, beber y hacer ejercicio parecen basarse en la premisa de 
que quienes reciben estos mensajes son grupos o comunidades homogéneos, integrados por "consumidores promedio"(75), evidenciando lo afirmado por Aguirre ${ }^{(76)}$ respecto de la visión que domina en las políticas de salud: que la obesidad es un problema significativo en los sectores de bajos ingresos al que se le ofrece soluciones que pueden Ilevar adelante los sectores de ingresos medios $\mathrm{o}$ altos. Por tanto, y aunque se ha reconocido hasta el cansancio, es necesario que las estrategias sobrepasen el ámbito del campo de la salud, dados los constreñimientos materiales que las personas enfrentan para modificar sus elecciones alimentarias o de práctica de actividad física.

La mayoría de las acciones, tomadas en su conjunto, se han remitido a traducir y aplicar las consignas globales más sencillas y rápidas de atender: en países socialmente desiguales es más fácil promover el consumo de alimentos saludables y el ejercicio físico regular que tratar de mitigar los determinantes sociales de la obesidad y/o modificar los factores estructurales que la condicionan. Sin embargo, durante el periodo estudiado, las políticas económicas han ido en sentido contrario, conllevando a una parte significativa de la población a situaciones de profunda precarización social y alimentaria. Como consecuencia de las crisis económicas vividas, las políticas de austeridad fiscal y monetaria impuestas por cada país, aunque en momentos diferentes, han conseguido aumentar la pobreza y dificultar el acceso a una alimentación saludable. Así, al analizar las acciones más relevantes en su conjunto, y a falta de evaluaciones específicas, se observa que en buena parte de ellas no se ha prestado la suficiente atención a los cambios socioeconómicos acontecidos en este periodo y cómo estos se han reflejado en las prácticas alimentarias. Asimismo, debemos hacer notar que Brasil y de manera similar Argentina, están viviendo un proceso de reconocimiento de la desnutrición y la obesidad como distintas expresiones de la inseguridad alimentaria, asumiendo con ello el enfoque sindémico que las vincula entre sí(77). Esta mayor desigualdad social en estos dos países podría explicar por qué el aumento de la obesidad, respecto a España, ha sido aun mayor. Pero, en los tres países, la obesidad constituye un epítome de la precarización, que cristaliza una forma particular de "cuerpos precarios". Por tanto el concepto de precarización remite a un conjunto de situaciones socioeconómicas y políticas complejas que exceden puestos de trabajos inseguros o coberturas sanitarias insuficientes. En tanto incertidumbre y exposición al peligro, abarca la totalidad de la existencia, el ambiente, los cuerpos y los modos de subjetivación ${ }^{(78)}$. Sin embargo, hasta la fecha no se ha comprendido en profundidad cómo los llamados "factores ambientales locales" influyen en las prácticas alimentarias y sus consecuencias, ya sea por la prontitud de la respuestas replicadas sin profundizar en la naturaleza y alcance del problema; ya sea porque directamente no se han realizado diagnósticos ajustados a cada contexto o ya sea por el desmantelamiento de las políticas previas que, como en el caso de Brasil, habían respondido de forma integrada a las diversas expresiones de la malnutrición, particularmente el hambre. Además, reconocemos los interesantes debates en España sobre la regulación de la publicidad de alimentos; la actual discusión, en Argentina, sobre el etiquetado frontal de advertencia nutricional; o los acuerdos con la industria alimentaria sobre reformulación de los alimentos en los tres países. Pero todos estos avances, no solo implican avanzar contra la resistencia de actores claves, como es el caso de ciertos sectores de la industria alimentaria ${ }^{(14)}$, sino que forman parte también de la monopolización conceptual que generaliza los modelos preventivos sobre la obesidad implementados en los tres países, borrando las singularidades que podrían asumir las estrategias locales. 


\section{AGRADECIMIENTOS}

El presente estudio forma parte de un proyecto de mayor alcance titulado La precarización de la vida cotidiana: (in)seguridad alimentaria, género y salud (CSO201674941, 2017-2019), financiado por el Ministerio de Educación, Cultura y Deporte, de España y cuya dirección estuvo a cargo de la Dra. Mabel Gracia Arnaiz.

\section{REFERENCIAS BIBLIOGRÁFICAS}

1. World Health Organization. Obesity: preventing and managing the global epidemic. Geneva: WHO; 2000.

2. Gracia Arnaiz M. Qué y cuánto comer: tomando medidas frente a las sociedades obesogénicas. Salud Colectiva. 2009;5(3):363-376. doi: 10.18294/sc.2009.240.

3. Legetic B. Globesidad, epidemia del siglo XXI. Medwave. 2004;4(11):e2578. doi: 10.5867/medwave.2004.11.2578.

4. Christiansen ML. "Homo Caloricus": La construcción epistemológica del estigma lipofóbico en los discursos mediáticos de salud pública. Salud Colectiva. 2018;14(3):623-637. doi: 10.18294/sc.2018.1602.

5. Swinburn B, Egger G, Raza F. Dissecting obesogenic environments: the development and application of a framework for identifying and prioritizing environmental interventions for obesity. Preventive Medicine. 1999;29:563-570. doi: 10.1006/pmed.1999.0585.

6. Bray G, Tartaglia L. Medicinal strategies in the treatment of obesity. Nature. 2000;404(6778):672-677. doi: $10.1038 / 35007544$.

7. Dinsa G. Obesity and socioeconomic status in developing countries: a systematic review. Obesity Reviews. 2012;13(11):1067-1079. doi: 10.1111/j.1467789X.2012.01017.x.

8. Ng M, Fleming $T$, Robinson $M$, Thomson B, Graetz $\mathrm{N}$, et al. Global, regional, and national prevalence of overweight and obesity in children and adults during 1980-2013: a systematic analysis for the Global Burden of Disease Study 2013. Lancet. 2014; 384(9945):766781. doi: 10.1016/S0140-6736(14)60460-8.

9. Dias P, Henriques P, Anjos L, Burlandy L. Obesidade e políticas públicas: concepções e estratégias adotadas pelo governo brasileiro. Cadernos de Saúde Pública. 2017;33(7): e00006016. doi: 10.1590/0102$311 \times 00006016$.

10. Popkin BM, Gordon-Larsen P. The nutrition transition: worldwide obesity dynamics and their determinants. International Journal of Obesity. 2004;28:S2-S9. doi: 10.1038/sj.ijo.0802804.

11. Swinburn B, Shelly A. Effects of TV time and other sedentary pursuits. International Journal of Obesity. 2008;32(Suppl 7):S132-S136. doi: 10.1038/ijo.2008.249.

12. Monteiro C, Bertazzi Levy R, Claro Moreira R, De Castro I, Cannon G. A new classification of foods based on the extent and purpose of their processing. Ca- dernos de Saúde Pública. 2010;26(11):2039-2049. doi: 10.1590/S0102-311X2010001100005.

13. Brownell K, Horgen K. Food fight: the inside story of the food industry, America's obesity crisis, and what we can do about it. New York: McGraw Hill Professional; 2003.

14. Piaggio L. El derecho a la alimentación en entornos obesogénicos: Reflexiones sobre el rol de los profesionales de la salud. Salud Colectiva. 2016;12(4):605-619. doi: 10.18294/sc.2016.934.

15. Gracia Arnaiz M. Taking measures in times of crisis: The political economy of obesity prevention in Spain. Food Policy. 2017;68:65-76. doi: 10.1016/j. foodpol.2017.01.001.

16. Jiwani SS, Carrillo-Larco RM, Hernández-Vásquez A, Barrientos-Gutiérrez T, Basto-Abreu A, Gutierrez L, et al. The shift of obesity burden by socioeconomic status between 1998 and 2017 in Latin America and the Caribbean: a cross-sectional series study. Lancet Global Health. 2019;7(12):E1644-E1654. doi: 10.1016/S2214109X (19)30421-8.

17. Hernáez Á, Zomeño MD, Dégano IR, Pérez-Fernández S, Goday A, Vila J, Civeira F, Moure R, Marrugat J. Excess weight in Spain: Current situation, projections for 2030, and estimated direct extra cost for the Spanish health system. Revista Española de Cardiología. 2019;72(11):916-924. doi: 10.1016/j.rec.2018.10.010.

18. World Health Organization. The Global Strategy on Diet, Physical Activity and Health. Washington: WHO; 2004.

19. Organización Panamericana de la Salud. Estrategia Regional y Plan de Acción para un Enfoque Integrado Sobre la Prevención y el Control de las Enfermedades Crónicas. Washington DC: OPS; 2007.

20. Agencia Española de Seguridad Alimentaria. Nutrición, Actividad Física y Obesidad (NAOS): Estrategia para la Nutrición, Actividad Física y Prevención de la Obesidad. Madrid: Agencia Española de Seguridad Alimentaria; 2005.

21. Ministerio de Salud de la Nación. Resolución 732/2016, Programa Nacional de Alimentación Saludable y Prevención de la Obesidad. Buenos Aires: MS; 2016.

22. Câmara Interministerial de Segurança Alimentar e Nutricional. Estratégia Intersetorial de Prevenção e Controle da Obesidade: recomendações para estados e municípios. Brasília DF: CAISAN; 2014.

23. Guest G. Applied thematic analysis. California: Sage Publications; 2012.

24. Kleinman J. The Illness Narratives. New York: Basic Books; 1988.

25. Fox A, Feng W, Asal V. What es driving global obesity trends?: Globalization or "modernization"? Globalization and Health. 2019;15(1):32. doi: 10.1186/ s12992-019-0457-y. 
26. World Health Organization. Population-based approaches to Childhood obesity prevention. Geneve: WHO; 2012.

27. World Health Organization. Obesity and inequalities. Copenhagen: WHO Regional Office for Europe; 2014.

28. World Health Organization. WHO European Region Food and Nutrition Action Plan 2014-2020. Copenhagen: WHO Regional Office for Europe; 2013.

29. Organización Mundial de la Salud. Declaración de Shanghái sobre la promoción de la salud en la Agenda 2030 para el Desarrollo Sostenible. Shangai: OMS; 2016.

30. Organización Panamericana de la Salud. Políticas y programas alimentarios para prevenir el sobrepeso y la obesidad: Lecciones aprendidas. Washington: FAO, OPS; 2018.

31. Varela-Moreiras $G$ et al. Obesidad y sedentarismo en el siglo XXI: ¿qué se puede y debe hacer? Nutrición Hospitalaria. 2013;28(Suppl 5):S1-S12.

32. Centro Nacional de Innovación e Investigación Educativa. Programa de alimentación, nutrición y gastronomía para educación infantil: El gusto es mío (PANGEI). Madrid: Subdirección General de Documentación y Publicaciones, AECOSAN; 2015

33. Generalitat de Catalunya, Departament de Salut. Pla Integral per a la promoció de la salut mitjançant l'activiat física i l'alimentació saludable. Barcelona: Generalitat de Catalunya; 2008.

34. Argentina. Ley 26396, Trastornos alimentarios [Internet]. 2008 [citado 22 sep 2019]. Disponible en: https://tinyurl.com/yxvxekcr.

35. Ministerio de Salud de la Nación. Boletín de vigilancia de enfermedades no transmisibles y factores de riesgo. Buenos Aires: MS; 2016.

36. Ministerio de Salud. Plan Nacional de Alimentación Saludable en la Infancia y Adolescencia para la Prevención del Sobrepeso y la Obesidad en Niños, Niñas y Adolescentes- Plan Así. Buenos Aires: MS; 2019.

37. Ministério da Saúde. Política Nacional de Alimentação e Nutrição. Brasília: MS; 2012.

38. Ministério da Saúde. Estratégias para o cuidado da pessoa com doença crônica: obesidade. Brasília: MS; 2014.

39. Rugani Ribeiro de Castro I. Obesidad: urge que avancen las políticas públicas para su prevención y control. Cadernos de Saúde Pública. 2017;33(7):e00100017. doi: 10.1590/0102-311X00100017.

40. Ministerio de Sanidad, Consumo y Bienestar Social. Encuesta Nacional de Salud. Madrid: Ministerio de Sanidad, Consumo y Bienestar Social; 2006.

41. Ministerio de Sanidad, Consumo y Bienestar Social. Encuesta Nacional de Salud. Madrid: Ministerio de Sanidad, Consumo y Bienestar Social; 2012.
42. Ministerio de Sanidad, Consumo y Bienestar Social. Encuesta Nacional de Salud. Madrid: Ministerio de Sanidad, Consumo y Bienestar Social; 2018.

43. Navarro V. Ataque a la democracia y al bienestar. Barcelona: Anagrama; 2015.

44. Instituto Nacional de Estadística. Encuesta de Población Activa. Madrid: INE; 2018.

45. Fernández D. Los salarios en la recuperación española. Cuadernos de Información Económica. 2017;260:1-12.

46. Peña-Casas R, Ghailani D, Spasova S, Vanhercke B. In-work poverty in Europe: A study of national policies. Brussels: European Commission; 2019.

47. Llanos Ortiz JC. El estado de la pobreza seguimiento del indicador de riesgo de pobreza y exclusión social en España 2008-2016., Madrid: EAPN España; 2017.

48. Cáritas Española. Memoria 2016 [Internet]. 2016 [citado 22 sep 2019]. Disponible en: https:/tinyurl.com/ y5hyr9xd.

49. Díaz Méndez C, García Espejo I, Otero Estévez S. Discursos sobre la escasez: estrategias de gestión de la privación alimentaria en tiempos de crisis. EMPIRIA, Revista de Metodología de Ciencias Sociales. 2018;40:85105.

50. Ministerio de Salud de la Nación. Encuesta Nacional de Factores de Riesgo para Enfermedades no Transmisibles. Buenos Aires: MS; 2018.

51. Ministerio de Salud de la Nación. Encuesta Nacional de Factores de Riesgo para Enfermedades no Transmisibles. Buenos Aires: MS; 2013.

52. Ministerio de Salud de la Nación. Encuesta Nacional de Factores de Riesgo para Enfermedades no Transmisibles. Buenos Aires: MS; 2009.

53. Ministerio de Salud de la Nación. Encuesta Nacional de Factores de Riesgo para Enfermedades no Transmisibles. Buenos Aires: MS; 2006.

54. Instituto Nacional de Estadística y Censos. Estadísticas sobre canasta básica alimentaria, Argentina: Año 2019 [Internet]. Buenos Aires: INDEC [citado 22 sep 2019]. Disponible en: https://tinyurl.com/y4wdfqc2.

55. Instituto Nacional de Estadística y Censos. Estadísticas sobre pobreza, Argentina: Año 2019 [Internet]. Buenos Aires: INDEC [citado 22 sep 2019] Disponible en: https://tinyurl.com/y2onvr5o.

56. Observatorio de la Deuda Social Argentina, Universidad Católica Argentina. Incidencia de la inseguridad alimentaria severa y total para los Hogares y la Población en la Argentina urbana 2010-2019 [Internet]. 2019 [citado 22 dic 2019]. Disponible en: https:/tinyurl.com/ yxrd66wk.

57. Aguirre P. Estrategias domésticas de consumo: qué comen los argentinos que comen. Buenos Aires: CIEPP; 2005. 
58. Instituto Brasileiro de Geografia e Estatística. Pesquisa de Orçamentos Familiares 2002-2003: análise da disponibilidade domiciliar de alimentos e do estado nutricional no Brasil. Rio de Janeiro: IBGE; 2004.

59. Instituto Brasileiro de Geografia e Estatística. Pesquisa de Orçamento Familiares 2008-2009: Antropometria e Estado Nutricional de Crianças, Adolescentes e Adultos no Brasil. Rio de Janeiro: IBGE; 2010.

60. Instituto Brasileiro de Geografia e Estatística. Pesquisa Nacional de Saúde, 2013. Rio de Janeiro: IBGE; 2014.

61. Ministério da Saúde. Vigilância de fatores de risco e proteção para doenças crônicas por inquérito telefônico Brasil 2018. Brasília: Ministério da Saúde; 2019.

62. Neri M. A escalada da desigualdade: qual foi o impacto da crise sobre a distribuição de renda e a pobreza? Rio de Janeiro: FGV Social; 2019.

63. España. Ley $17 / 2011$, Seguridad alimentaria y nutrición [Internet]. 2011 [citado 22 sep 2019]. Disponible en: https://tinyurl.com/y4jz2vm8.

64. Ballesteros Arribas JM, Pérez Farinós N, Quiles i Izquierdo J, Echeverría Cubillas P, Castell i Abat C, Muñoz Bellerín J, et al. Evaluación y seguimiento de la Estrategia NAOS: conjunto mínimo de indicadores. Madrid: Ministerio de Sanidad, Política Social e Igualdad; 2011.

65. Eduard Ortega-Rodríguez E, Sánchez-Martínez F, Valmayor S, Juárez O, Pasarín I, Grupo de Investigación de Proyecto POIBA. La prevención de la obesidad infantil desde una perspectiva comunitaria. Atención Primaria. 2014;47(4):246-255. doi: 10.1016/j.aprim.2014.11.006.

66. Sánchez-Martínez F, Torres Capcha P, Serral Cano G, Valmayor Sanfont S, Castell Abat C, Ariza Cardenal C, Grupo de evaluación del Proyecto POIBA. Factores asociados al sobrepeso y la obesidad en escolares de 8 a 9 años de Barcelona. Revista Española de Salud Pública. 2016;90:e40027.

67. Ministerio de Salud de la Nación. Proyecto de Protección de la población vulnerable contra las enfermedades crónicas no transmisibles. Buenos Aires: MS; 2015.

68. Ministerio de Salud de la Nación. Sobrepeso y Obesidad en Argentina. [Internet]. 2017 [citado 22 sep 2019]. Disponible en: https://tinyurl.com/yxzpbart.
69. Triches R, Schneider S. Desestruturar para construir: interfaces para a agricultura familiar acessar o programa de alimentação escolar. Estudos Sociedade e Agricultura. 2012;20(1):66-105.

70. Ministério da Saúde. Guia alimentar para a população brasileira. Brasília: MS; 2014.

71. Backes V, Bairros F, Cafruni CB, Cummins S, ShareckM, Mason K, et al. Food environment, income and obesity: a multilevel analysis of a reality of women in Southern Brazil. Cadernos de Saúde Pública. 2019;35(8):e00144618. doi: 10.1590/0102-311x00144618.

72. Lopes A, Menezes M, Araújo M. O ambiente alimentar e o acesso a frutas e hortaliças: "Uma metrópole em perspectiva". Saúde e Sociedade. 2017; 26(3): 764 773. doi: 10.1590/s0104-12902017168867.

73. Camargo D, Belon A, Marín-León L, Souza BF Pérez-Escamilla R, Segall-Corrêa A. Comparing food environment and food purchase in areas with low and high prevalence of obesity: data from a mapping, in-store audit, and population-based survey. Cadernos de Saúde Pública. 2019;35(9):e00247218. doi: 10.1590/0102$311 \times 00247218$

74. Centro de Investigación sobre Problemáticas Alimentarias Nutricionales, Fundación Interamericana del Corazón, Programa Nutricional de la Ciudad de Buenos Aires. Estudio multicêntrico sobre entornos obesogénicos en situaciones de vulnerabilidad: Presentación de resultados. Ciudad de Buenos Aires: CIPAN, FIC; 2019.

75. Monagahn LF, Colls R, Evans B. Obesity discourse and fat politics: research, critique and interventions. Critical Public Health. 2013;23(3):249-262. doi: 10.1080/ 09581596.2013 .814312$.

76. Aguirre P. Una historia social de la comida. Buenos Aires: Lugar Editorial; 2017.

77. Swinburn BA, Kraak VI, Allender S, Atkins VJ, Baker PI, Bogard JR, et al. The global syndemic of obesity, undernutrition, and climate change: The Lancet commission report. Lancet. 2019;393:791-846. doi: 10.1016/S0140-6736(18)32822-8.

78. Romaní O, Casadó L (eds.). Jóvenes, desigualdades y salud: Vulnerabilidad y políticas públicas. Tarragona: URV; 2014

\section{FORMA DE CITAR}

Gracia Arnaiz M, Demonte F, Kraemer FB. Prevenir la obesidad en contextos de precarización: respuestas locales a estrategias globales. Salud Colectiva. 2020;16:e2838. doi: 10.18294/sc.2020.2838.

Recibido: 8 may 2020 | Aprobado: 6 oct 2020 | Publicado en línea: 10 nov 2020

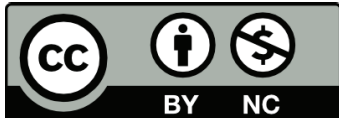

Esta obra está bajo una licencia de Creative Commons Reconocimiento-NoComercial 4.0 nternacional. Reconocimiento - Permite copiar, distribuir y comunicar públicamente la obra. A cambio, se debe reconocer y citar al autor original. No Comercial - Esta obra no puede ser utilizada con finalidades comerciales, a menos que se obtenga el permiso. 